\title{
Effects of gravitational forces on single joint arm movements in humans
}

Received: 31 January 1993/Accepted: 29 November 1993

\begin{abstract}
We have examined the kinematics and muscle activation patterns of single joint elbow movements made in the vertical plane. Movements of different amplitudes were performed during a visual, step-tracking task. By adjusting shoulder position, both elbow flexion and extension movements were made under three conditions: (a) in the horizontal plane, (b) in the vertical plane against gravity, and (c) in the vertical plane with gravity. Regardless of the gravitational load, all movements were characterized by time symmetric velocity profiles. In addition, no differences were found in the relationships between movement duration, peak velocity, and movement amplitude in movements with or against gravity. The pattern of muscle activation was influenced however, by the gravitational load. Both flexion and extension movements made with gravity were characterized by a reciprocally organized pattern of muscle activity in which phasic agonist activity was followed by phasic antagonist activity. Flexion and extension movements made against gravity were characterized by early phasic antagonist activity occurring at about the same time as the initial agonist burst. These findings suggest that EMG patterns are modified in order to preserve a common temporal structure in the face of different gravitational loads.
\end{abstract}

Key words Voluntary movement $\cdot$ Kinematics $\cdot$ EMG Gravitational loading · Human

N. Virji-Babul · J.D. Cooke $(\bowtie) \cdot$ S. H. Brown ${ }^{1}$ Faculty of Applied Health Sciences, Elborn College, University of Western Ontario, London, Ontario, Canada N6G 1H1

\footnotetext{
Present address:

${ }^{1}$ Deptartment of Movement Science, University of Michigan, Ann Arbor, MI 48109-2214, USA
}

\section{Introduction}

The control strategies used by the CNS in generating skilled movement continues to be one of the fundamental questions in motor control. A major focus of research has been directed towards describing the relationship between specific kinematic variables and the underlying muscle activation patterns in order to identify regularities in the coordination of limb movement. To this end, many studies in the past have been restricted to single joint movements in the horizontal plane. Such movements, however, comprise only a small part of our 'natural' movement repertoire. Many movements are made in the vertical plane, where a number of complexities arise due to the influence of gravitational forces. For example, the magnitude of the gravitational load does not remain constant but changes with joint angle. Furthermore, gravitational loads pose different demands for the motor system depending on the direction in which the movement is made, i.e., elbow flexions are commonly made against gravity, while elbow extensions are made with gravity. How does the CNS organize such movements and how does this organization compare or relate to the known properties of movements made in the horizontal plane?

Many single joint movements made in the horizontal plane are characterized by a smooth, bell-shaped velocity profile in which the duration of the acceleration and deceleration phases are approximately equal (Ostry et al. 1987). Although the velocity profile may be temporally asymmetric in movements requiring a high degree of accuracy (Soechting 1984; Gentilucci et al. 1991), time symmetric velocity profiles have been reported for many well-learned movements, including single joint movements in the vertical plane (Atkeson and Hollerbach 1985), multijoint movements (Morasso 1981; Soechting 1984; Kaminski and Gentile 1986), speech movements (Ostry 1986) and movements of the vocal folds (Munhall et al. 1985). Time-symmetric velocity profiles have been found to remain consistent under transformations of movement amplitude, duration, 
speed, and load (Morasso 1981; Atkeson and Hollerbach 1985; Ostry et al. 1987; Cooke et al. 1989) leading to the suggestion that this profile may represent a fundamental organizing principle underlying movement through minimization of energy (Nelson 1983), optimization of joint stiffness (Hasan 1986), or minimizing the rate of change of acceleration (i.e., jerk) (Hogan 1984).

How does the nervous system formulate the motor commands to produce movements having a common temporal structure? Movements of different temporal structures have been shown to be produced by modification of a 'triphasic' pattern of muscle activation (Brown and Cooke 1990). Components of the triphasic pattern are highly correlated with specific kinematic parameters. For example, both the magnitude and duration of the initial agonist burst (AG1) increase with movement amplitude (Berardelli et al. 1984; Brown and Cooke 1984; Benecke et al. 1985). How do the EMGmovement relationships observed in horizontal movements compare to movements made under the influence of gravity? To date, few studies have examined both the kinematics and the underlying muscle activation patterns for movements in this plane. Cheron and Godaux (1986) reported that elbow flexion movements made in the vertical plane were characterized by a 'triphasic' pattern of muscle activation, similar to that observed in movements made in the horizontal plane. However, Stein et al. (1988) demonstrated that the pattern of muscle activity was in fact highly influenced by loading conditions. They examined wrist flexion movements performed under elastic, viscous, and inertial loads. Each loading condition was associated with a specific pattern of muscle activity resulting in the production of quite similar movements. Given the inconsistent data on the effects of gravitational and other loads, our purpose was to examine and compare in more detail, the EMGmovement relationship in single joint movements made in both the horizontal and vertical planes. The data to be presented here demonstrate that regardless of the gravitational load, movements are characterized by time symmetric velocity profiles. This profile is associated with modification of a basic pattern of muscle activation which is dependent on whether movements are performed with or against gravity.

\section{Methods}

Experimental paradigm

Eight normal subjects (aged 22-52 years) with no known history of motor system disorders participated in this study. Subjects performed elbow flexion and extension movements in a visual steptracking paradigm. The subject's forearm position was displayed as a horizontal line on a television monitor placed at eye level $1.8 \mathrm{~m}$ in front of the subject. A horizontal target bar displayed on the screen switched at a regular rate (every $5 \mathrm{~s}$ ) between two fixed vertical positions. Subjects were required to superimpose the position cursor on the target bar and were instructed to move "fast and accurately." By adjusting the shoulder position, elbow flexion and extension movements were made under the following three conditions:

\section{Vertical plane: flexion against gravity/extension with gravity}

Each subject was seated comfortably with the shoulder in 0 deg abduction, elbow flexed to $100 \mathrm{deg}$ (full elbow extension $=180$ $\mathrm{deg}$ ), forearm supinated and fingers lightly flexed. Movements of five different amplitudes $(5,10,20,30$, and $40 \mathrm{deg})$ were performed from this starting position. At each amplitude a block of 30 movements consisting of 15 flexion and 15 extension movements was performed. Presentation of each new block was preceded by a rest period of 2-3 min. Several practice movements were made at each amplitude prior to data collection.

\section{Vertical plane: flexion with gravity/extension against gravity}

In three subjects the direction of the gravitational load was reversed. Each subject was seated with the shoulder flexed to 180 deg, elbow flexed to $100 \mathrm{deg}$, forearm supinated, and fingers lightly flexed. In this position, $30 \mathrm{deg}$ elbow flexion movements were made with gravity while extension movements were made against gravity. Each experimental session consisted of two blocks of movements, each block consisting of a total of 15 movements (eight flexion and seven extension). The number of trials in each block was reduced in this condition in order to reduce fatigue resulting from maintaining the shoulder in this position for prolonged periods of time.

\section{Horizontal plane}

Each subject was seated comfortably and grasped a vertical rod attached to a manipulandum which rotated in the horizontal plane about a vertical axis. The subject's shoulder was abducted to 90 deg with the elbow flexed to $100 \mathrm{deg}$ and supported beneath the pivot point. Thirty elbow movements at an amplitude of 20 deg were performed in this position.

\section{Data recording}

Angular positions for movements made in the vertical plane were obtained using an electrogoniometer (Penny and Giles). For movements in the horizontal plane, the angular position of the manipulandum (and thus the elbow joint) was measured with a precision potentiometer. Surface EMGs were recorded from the biceps and lateral head of triceps brachii with $\mathrm{Ag}-\mathrm{AgCl}$ electrodes $(0.8 \mathrm{~cm}$ in diameter) placed longitudinally about $3 \mathrm{~cm}$ apart over the muscle bellies. EMGs were filtered $(10-1000 \mathrm{~Hz}$ bandpass $)$ and full wave rectified prior to digitization. For movements made in the horizontal plane, angular position was obtained from a precision potentiometer. The data were digitized on-line at $500 \mathrm{~Hz}$, and stored for later off-line analysis.

\section{Data analysis}

Kinematic data were smoothed by digital filtering $(30 \mathrm{~Hz}$, zero phase shift) prior to analysis. Velocity and acceleration were obtained from individual flexion and extension movements by differentiation of the position signal. The times of the start and end of acceleration and deceleration were determined using a threshold of $120 \mathrm{deg} / \mathrm{s}^{2}$. These times were used in determining movement duration, peak velocity and symmetry ratio (i.e., the ratio of acceleration duration to deceleration duration). Mean values for peak velocity, movement duration and acceleration/deceleration duration ratios from each subject were used to calculate the means and standard deviations across all subjects, at each amplitude. Onset and offset times of EMG bursts were determined using interactive 
Fig. 1 Movements made with and against gravity. Records of position and velocity from extension (with gravity, upper set) and flexion (against gravity, lower set) are shown for movements of three amplitudes $(10,20$, and $40 \mathrm{deg})$. Each record is the average of 15 movements. Dashed lines indicate $\pm 1 \mathrm{SD}$. Records were aligned to movement start for averaging
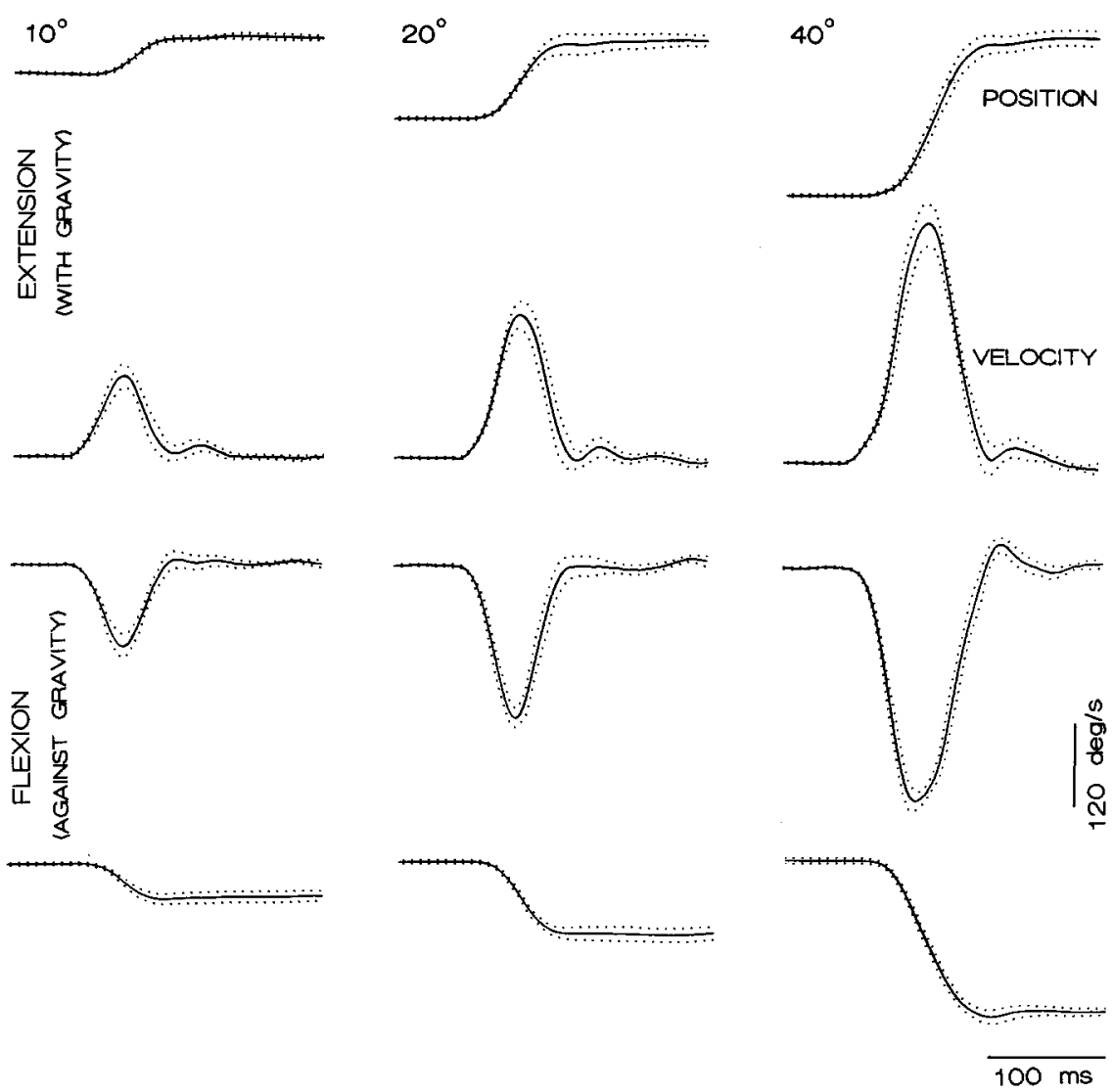

graphics. Only those records in which EMG onset and offsets could be clearly identified were used for analysis. As a result records from only three subjects were used in this analysis (shown in Fig. 6).

\section{Moments of force}

Since the EMG activity must, in some way, reflect the force output of the muscles, we analyzed the torques acting on the limb using the following equation governing the motion of a single segment:

$I \alpha=T m-m g r \cos \theta$

where

$I=$ moment of inertia of the segment, $\alpha=$ angular acceleration, $T m=$ moment of force (torque) due to muscle activity, $m=$ mass of the segment (forearm plus hand), $g=$ gravitational acceleration, $r=$ distance from center of gravity to the pivot point, $\theta=$ segment angle

This equation can also be written as:

$T e=T m-T g$

where

$T e=$ net torque resulting in angular acceleration of the limb, $T m=$ torque due to muscle activity, $T g=$ torque due to gravitational acceleration

\section{Results}

Movement kinematics

Averaged position and velocity records of extension (with gravity) and flexion (against gravity) movements made in the vertical plane by one representative subject are shown in Fig. 1. The characteristic bell-shaped velocity profile was observed at all amplitudes for both flexion and extension movements. In several subjects, extension movements made with gravity did not terminate smoothly and a small period of oscillation was observed at the end of movement. In flexion movements, this was only observed in large amplitude movements.

Changes in kinematic parameters with movement amplitude across six subjects are shown in Fig. 2. Peak velocity increased linearly with movement amplitude for both flexion $(r=0.99)$ and extension $(r=0.99)$ movements (Fig. 2A). There was no significant difference in the slopes $(P=0.15)$. In addition, no significant difference was found in the peak velocities between flexions and extensions at any amplitude (e.g., for $40 \mathrm{deg}$ amp: $P=0.43$ ). Figure $2 \mathrm{~B}$ illustrates the relation between movement duration and movement amplitude. Movement duration increased linearly with movement amplitude for both flexion and extension (flexion $r=0.97$, extension $r=0.96$ ). On average, extension (with gravity) movements appeared to be of shorter duration than flexion (against gravity) movements. However, no signif- 


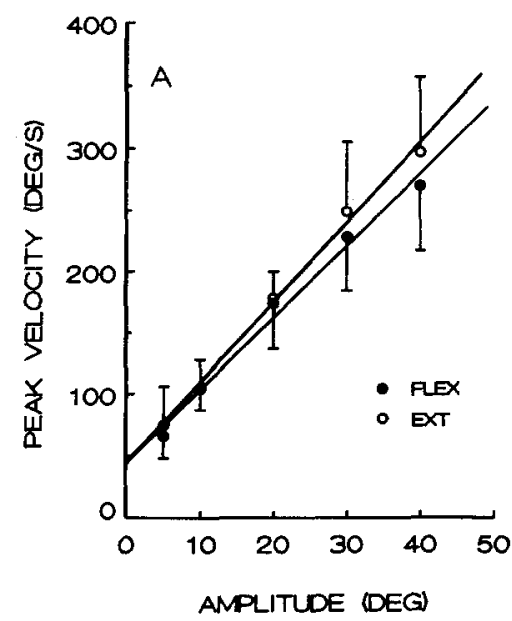

Fig. 2 Kinematic relations. Graphs of peak velocity (A), movement duration $(\mathbf{B})$ and the ratio of acceleration to deceleration durations (C) are shown. Each point is the average ( $\pm 1 \mathrm{SD})$ obtained from six subjects. Solid lines are the best-fit linear regression lines for data from flexions (closed symbols) and extensions (open symbols). A Peak velocity/amplitude: flexion $\mathrm{Vp}=45+5.9$ $\mathrm{A}$; extension $\mathrm{Vp}=44+6.5 \mathrm{~A}$. B Movement duration/amplitude: flexion $\mathrm{MD}=196+3.1 \mathrm{~A}, r=0.97$; extension $\mathrm{MD}=177+2.9 \mathrm{~A}$, $r=0.96$. C Symmetry ratio/amplitude: flexion $\mathrm{SR}=0.88-0.003 \mathrm{~A}$, $r=0.71$; extension $\mathrm{SR}=0.86+0.004 \mathrm{~A}, r=0.76$. The dashed horizontal line in $\mathrm{C}$ indicates a ratio of 1.0

icant differences were found between the slopes $(P=0.37)$ or between movement durations at any amplitude (for 40 deg amp: $P=0.23$ ).

Mean symmetry ratios (acceleration duration/deceleration duration) are plotted in Fig. $2 \mathrm{C}$ for six subjects. A significant difference was found between the slopes of the two regression lines $(P<0.05)$. For flexion movements there was a trend towards decreasing symmetry ratios as amplitude increased. Symmetry ratios ranged from 0.8 to 0.9 with a mean of 0.82 indicating that, on average, the duration of deceleration was slightly greater than the duration of acceleration for movements made against gravity. Extension movements made with gravity exhibited a trend towards increasing symmetry ratios as amplitude increased. Symmetry ratios ranged from $0.8-1.2$, with a mean of 0.9 . Statistical analysis, however, revealed a significant difference only for $30 \mathrm{deg}$ movements $(P=0.006)$. This difference may have arisen in part from the data of one particular subject who had consistently larger symmetry ratios for extension and smaller ratios for flexions at the larger amplitudes. If this subject's data were removed, no significant differences remained. In general extension movements were time symmetric at all amplitudes. Flexion movements exhibited slightly asymmetric profiles, with deceleration duration marginally greater than acceleration duration.

In order to further compare flexion and extension movement profiles, averaged velocity records were adjusted for movement duration and peak velocity. Averaged flexion records for each amplitude were used as a reference. The averaged peak velocities and movement
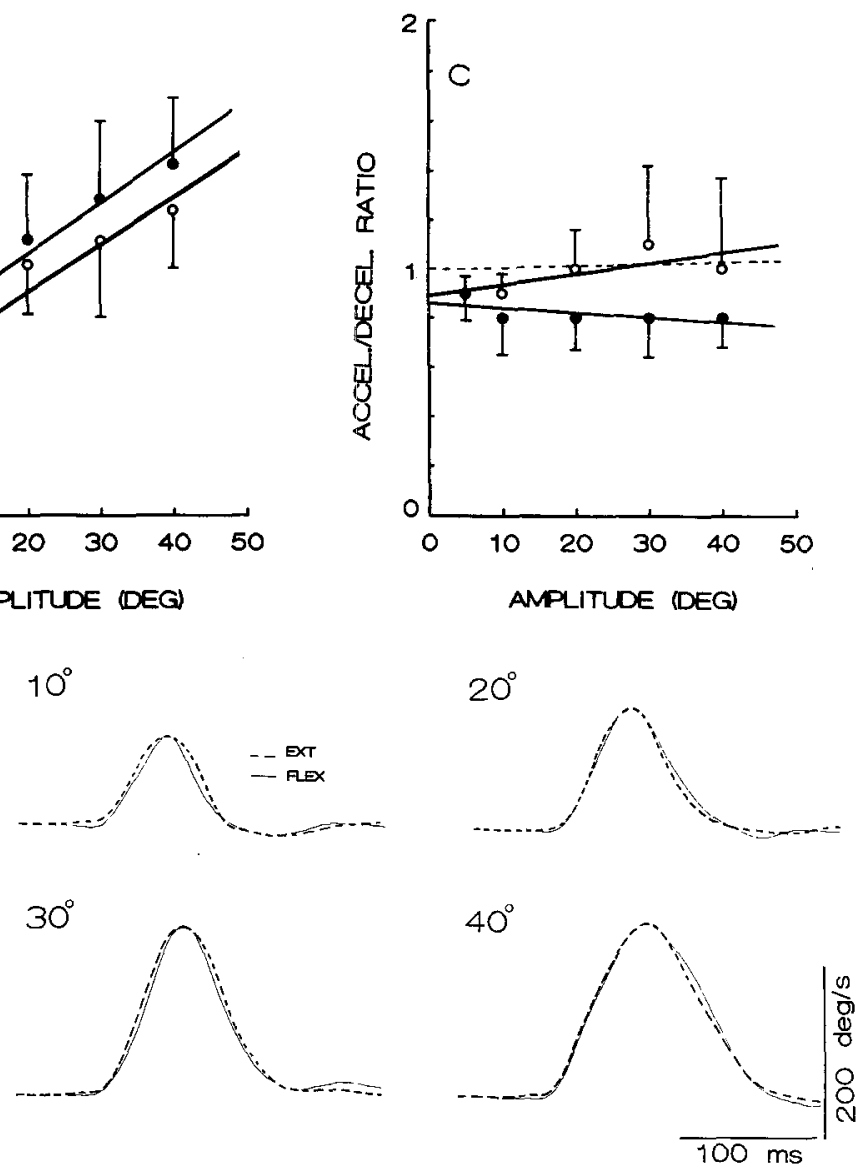

Fig. 3 Scaling of velocity profiles. Averaged velocity records are shown from movements of four amplitudes (10, 20, 30 and $40 \mathrm{deg})$ made by one subject. Each record is the average of 15 movements. At each movement amplitude, the average velocity records from extension movements (dashed lines) were scaled to the duration and peak velocity of the corresponding flexion movements (solid lines) and inverted for plotting. Records were aligned to movement onset for averaging and plotting

durations from extension movements were scaled by an appropriate factor and overplotted on the flexion records. Figure 3 illustrates the velocity profiles from one subject. This figure illustrates an overlap in the velocity profiles between the two different movements at all amplitudes, demonstrating that a common movement profile was produced for movements with and against gravity.

\section{Muscle activation patterns/moments of force}

\section{Horizontal plane}

In Fig. 4 the averaged position, velocity, and associated EMGs are shown for flexion and extension movements made in the horizontal plane. Both flexion and extension movements were time-symmetric and in both, AG1 occurred prior to movement start, providing the driving force to set the limb in motion. Phasic activity in ANT 
Fig. 4 EMG activity during single joint elbow flexion and extension movements made in the horizontal plane. Averaged position and velocity records from flexion and extension movements in the horizontal plane. Records are the average of 15 movements for 20 deg movements. Records were aligned to movement start for averaging. Dotted line indicates movement onset. Vertical position and velocity calibration represents $10 \mathrm{deg}$ and $130 \mathrm{deg} / \mathrm{s} \mathrm{re-}$ spectively
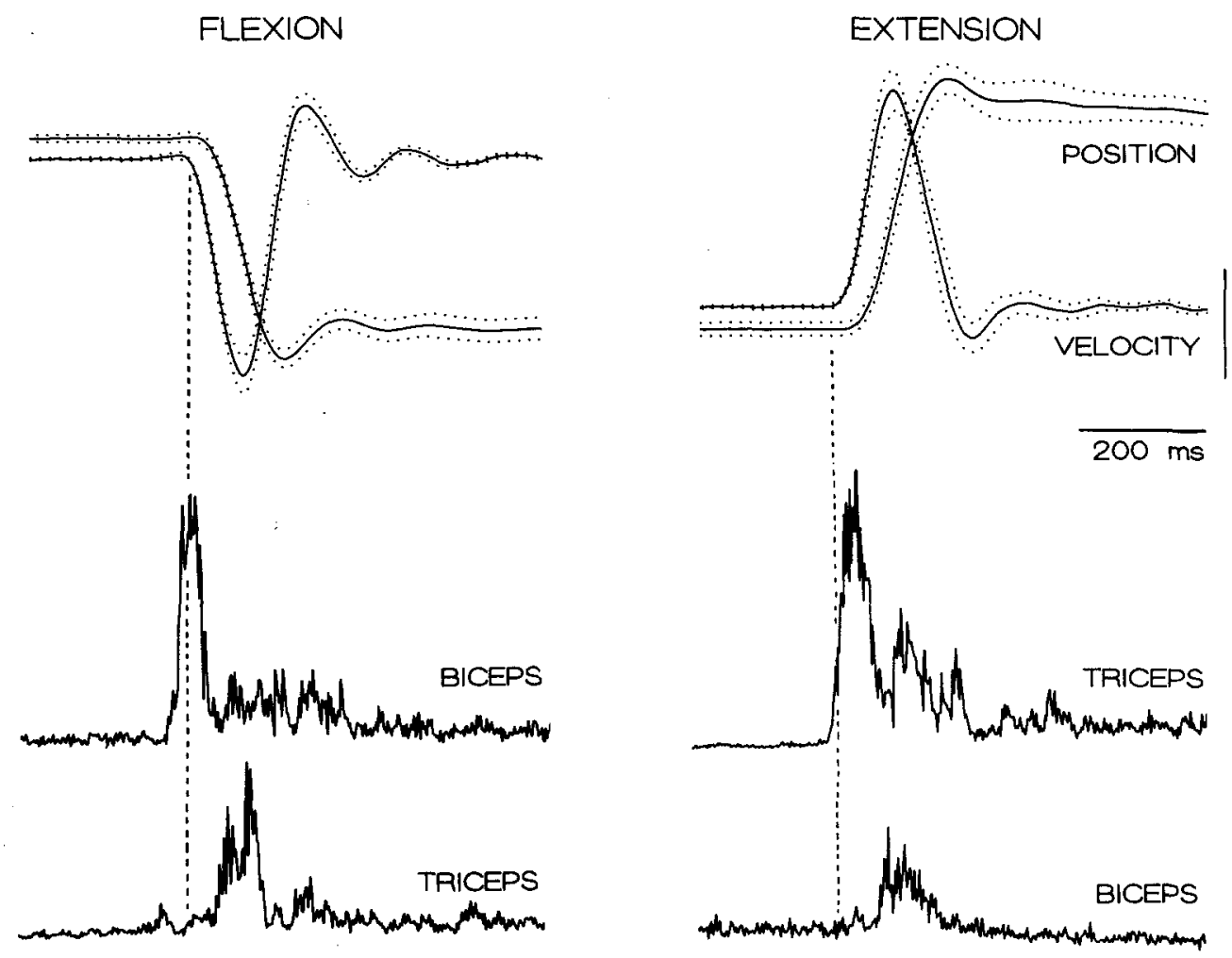

occurred at or near peak velocity and was followed by a second, smaller burst in the agonist (AG2).

\section{Vertical plane-flexion against gravity/extension with gravity}

The muscle activation patterns associated with flexion (upper panel) and extension (lower panel) movements made in the vertical plane are illustrated in Fig. 5. Both flexion and extension movements were initiated by AG1 which occurred prior to movement start. The data from this subject shows that AG1 occurred earlier (with respect to movement onset) for movements made with gravity in comparison to movements made against gravity; this was not, however, a consistent finding across all subjects. The most striking difference between flexion and extension movements was related to the time of onset of phasic antagonist activity. For flexion movements made against gravity, phasic antagonist activity started at virtually the same time as AG1. A second antagonist burst followed at about the time of movement peak velocity. In contrast, in extension movements made with gravity, a single antagonist burst occurred later in the movement, near the time of peak velocity.

A linear relationship was observed between AG1 duration and movement amplitude in both flexion and extension movements. However, differences were observed in the slopes of this relation. The data in Fig. 6 represent averaged burst durations taken from individual records from three subjects. For flexion (against gravity) movements the mean burst durations ranged from $57( \pm 1)$ $\mathrm{ms}$ for $10 \mathrm{deg}$ movements to $112( \pm 3) \mathrm{ms}$ for $40 \mathrm{deg}$ movements. For extension (with gravity) movements burst durations ranged from $48( \pm 9) \mathrm{ms}$ for $10 \mathrm{deg}$ movements to $62( \pm 11) \mathrm{ms}$ for $40 \mathrm{deg}$ movements. Although in both flexion and extension movements AG1 duration increased linearly with movement amplitude (flexion: $r=0.99$; extension: $r=0.92$ ), the slope of the relation was greater in flexion movements. A significant difference was found between the slopes of the two regression lines $(P<0.001)$. The right hand graph in Fig. 6 shows the time of onset of (ANT) relative to the onset of (AG1), for both flexion (against gravity) and extension (with gravity) movements. In flexions, ANT onset occurred on the average, $12( \pm 4.6) \mathrm{ms}$ after the onset of AG1. Since the shortest agonist burst duration was 57 $( \pm 1) \mathrm{ms}$, this indicates that phasic activity in the agonist and antagonist occurred quite close together. In contrast, during extension movements, ANT onset occurred on the average, $91.5( \pm 22.5) \mathrm{ms}$ after the onset of AG1. The largest mean AG1 burst duration in this case was $62( \pm 11) \mathrm{ms}$. Thus, there was little if any AG1-ANT coactivity during extension movements.

Figure 7 shows the torques due to gravity $(T g)$, muscle activity $(T m)$ and the net torque (Te) calculated from the averaged data of one representative subject for a movement amplitude of $30 \mathrm{deg}$. By convention, forearm movement in a counterclockwise direction was considered to be positive and those in a clockwise direction negative. In both flexion (against gravity) and extension (with gravity) movements, the magnitude of the static torque (prior to movement start) due to gravity $(T g)$ was approximately $1.7 \mathrm{Nm}$, acting in a negative direction to 

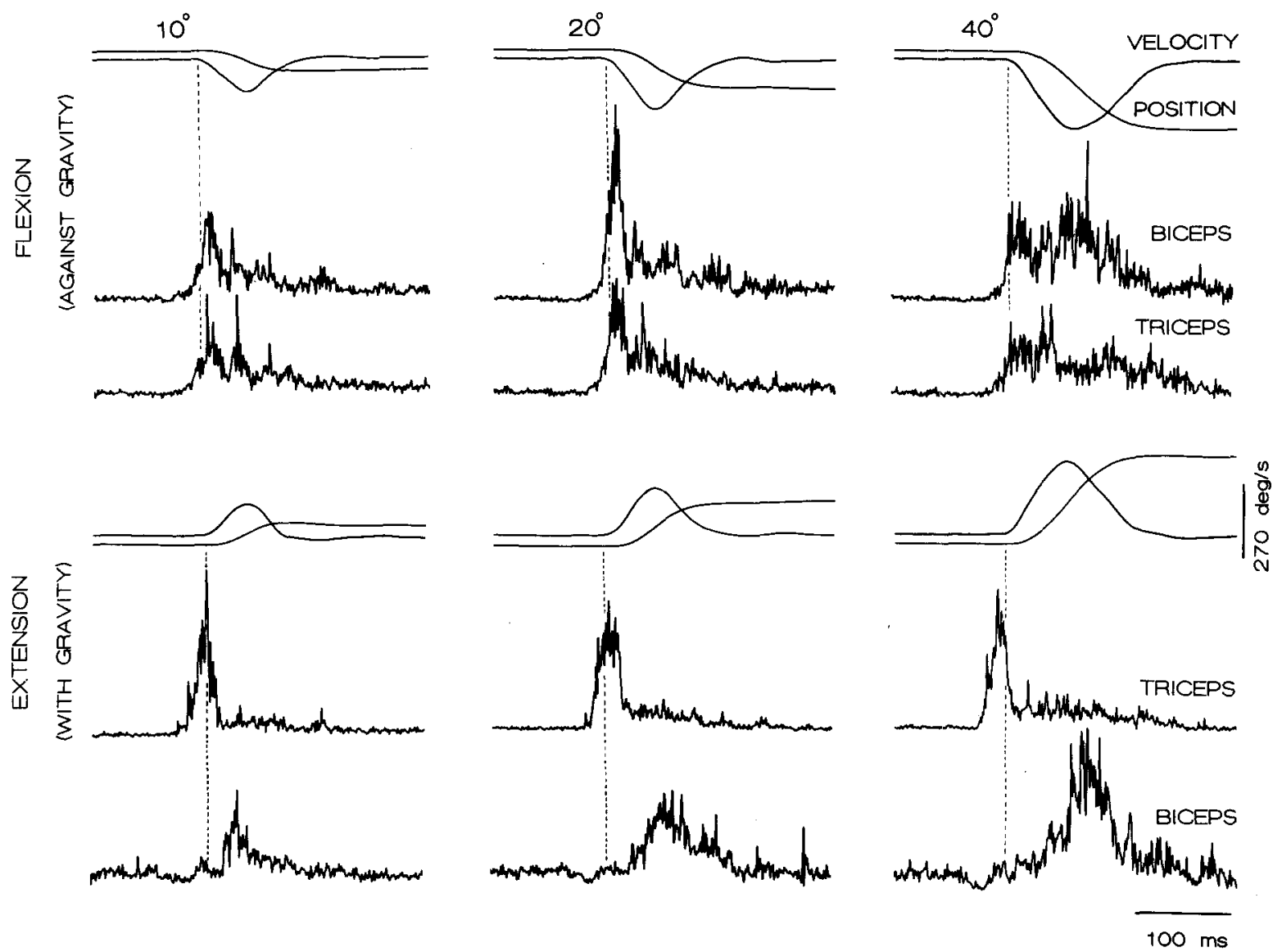

Fig. 5 EMG activity during elbow movements made in the vertical plane. In each set of records are shown averaged position, velocity and EMG activity for movements of three amplitudes $(10,20$, and $40 \mathrm{deg})$. Traces are the average of 15 movements. The upper set illustrates flexion (against gravity) movements and the lower set illustrates extension (with gravity) movements. Records were aligned to movement start for averaging. Vertical dotted line indicates movement onset. It should be noted that the amplitude scale across all panels is constant
Fig. 6 AG1 Duration and onset latency of ANT. The change in AG1 duration with amplitude is shown in the left hand graph. Each point is the average $( \pm 1$ SD) obtained from three subjects. Solid lines are the best-fit linear regression lines for data from flexions (closed symbols) and extensions (open symbols). The right hand graph shows the time span between the onset of AG1 and ANT. Each bar is the average $( \pm 1 \mathrm{SD})$ from three subjects. Flexions are indicated by open bars and extensions by striped bars

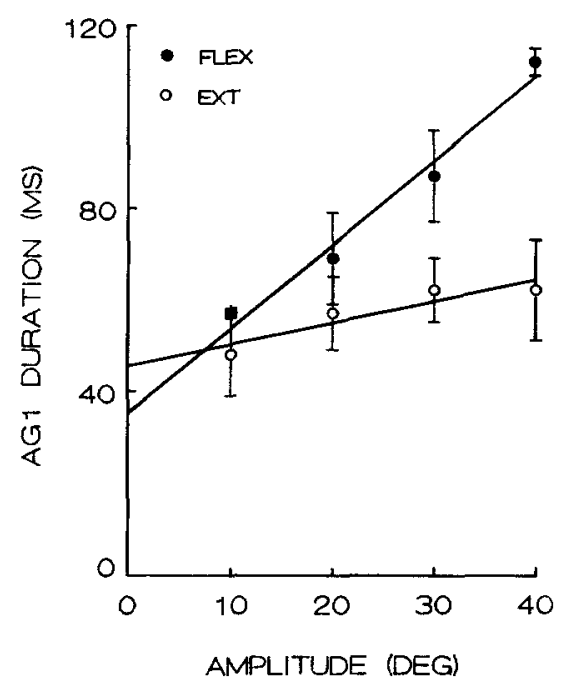

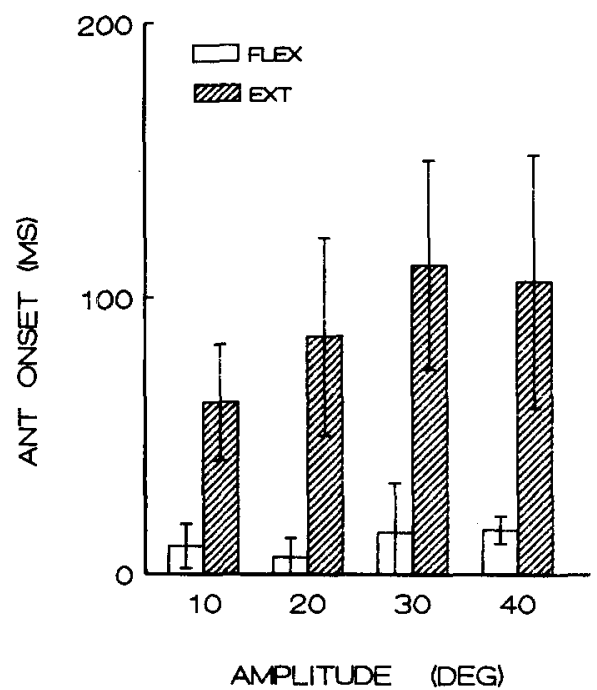

produce a clockwise rotation of the forearm. Note that there was little change in the magnitude of $\mathrm{Tg}$ throughout both flexion and extension movements. In flexion (against gravity) movements, $T g$ was acting in a direction opposite to the intended movement (Fig. 7, left panel). The magnitude of $T m$ was $1.7 \mathrm{Nm}$ prior to movement start and increased to $2.7 \mathrm{Nm}$ at the time of peak torque. The net change in magnitude and direction of Tm was due to two distinct components. The first was the change in torque needed to overcome the inertial 
Fig. 7 Calculated torques during movement. Records of the torque due to gravity $(T g)$, torque due to muscle activity $(T m)$ and net torque (Te) for flexion (against gravity) and extension (with gravity) movements. Traces are the average of ten movements for $30 \mathrm{deg}$ movements
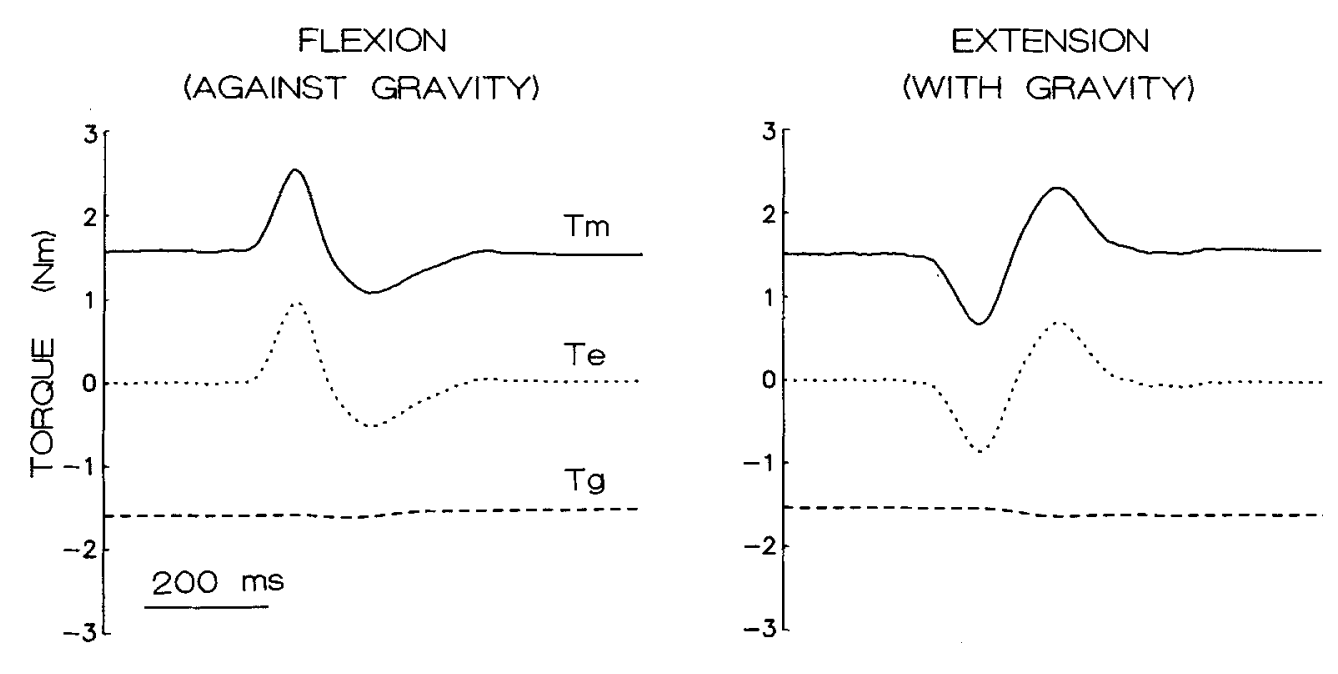

Fig. 8 Torques and EMGs during reversed loading conditions. Records of the torque due to gravity $(T g)$, torque due to muscle activity $(\mathrm{Tm})$ and net torque ( $\mathrm{Te}$ ) for flexion (with gravity) and extension (against gravity) movements. In each set of records are shown the averaged position, velocity and EMG activity for 30 deg movements. Traces are the average of ten movements. Records were aligned to movement start for averaging

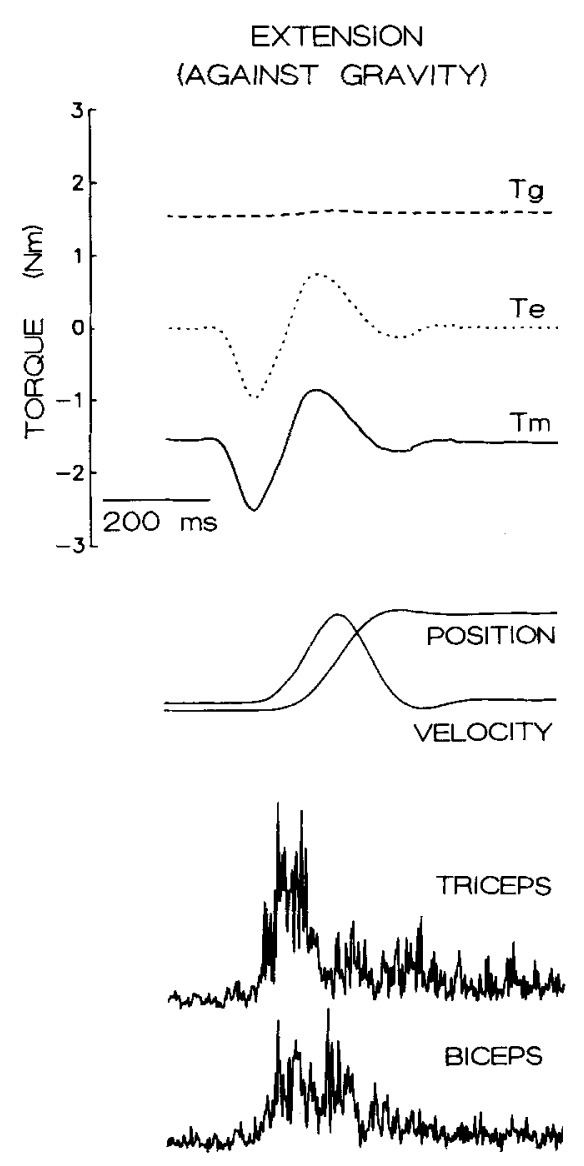

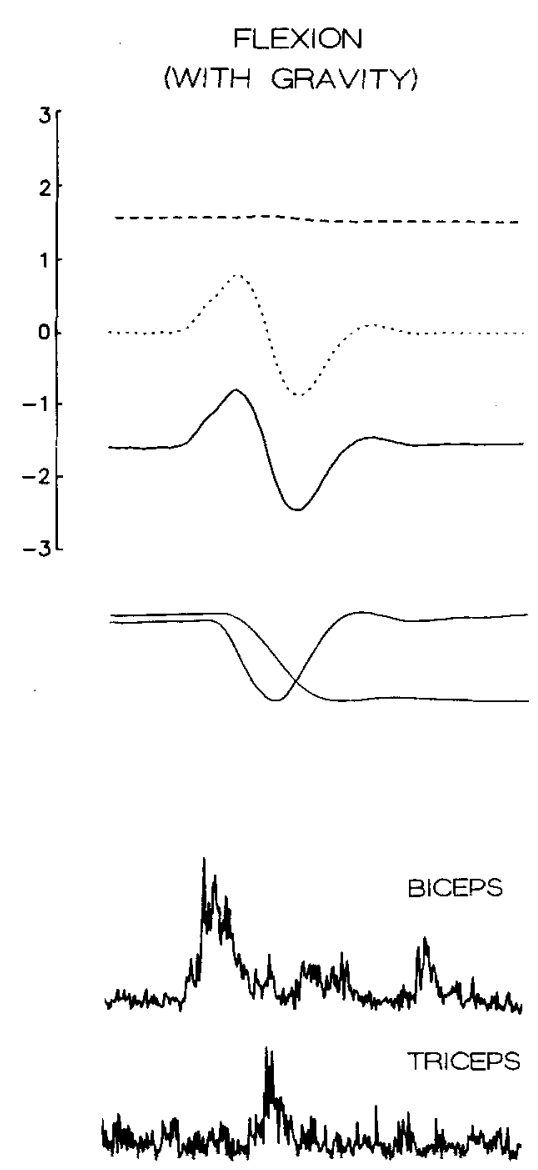

forces: this was proportional to acceleration. The second was the flexor torque required to compensate for $\mathrm{Tg}$. Since there was little change in the magnitude of $T g$ during the movement, the component of Tm necessary to overcome $\mathrm{Tg}$ was held approximately constant as movement progressed. That is, the presence of $\mathrm{Tg}$ served to increase the baseline magnitude of $T m$. The right panel in Fig. 7 illustrates that in extension movements made with gravity, $T g$ was acting in the same direction as the intended limb movement. The magnitude of $T m$ was
$1.5 \mathrm{Nm}$ prior to movement start and decreased to $0.7 \mathrm{Nm}$ at the time of peak torque. Again, the magnitude and direction of Tm was based on the torque necessary to overcome the inertial forces and to account for the presence of $T g$. In this case, however, $\mathrm{Tg}$ served to assist limb acceleration, resulting in a decrease in the magnitude of $T m$ as the movement progressed. As illustrated, an increase in flexor torque was required to overcome the clockwise torque due to $T g$ and bring the limb to a stop. 
Vertical plane-flexion with gravity/extension against gravity

The reversal in the pattern of the gravitational load resulted in an appropriate modulation of the muscle activation patterns. Figure 8 illustrates the averaged position and velocity records and the torques due to gravity $(T g)$, muscle contraction $(T \mathrm{~m})$, and net torque producing angular rotation ( $T e$ ) for flexion (with gravity) and extension (against gravity) movements. The corresponding averaged EMG muscle activation patterns are also shown. In this task $\mathrm{Tg}$ was acting to produce a positive or counterclockwise rotation of the forearm. Thus, in flexion movements made with gravity, $\mathrm{Tg}$ was acting in the same direction as the limb movement (right hand graph in Fig. 8). A static extensor torque of $-1.4 \mathrm{Nm}$ was produced prior to movement start and decreased to $-0.8 \mathrm{Nm}$ at the time of peak force. In extension (against gravity) movements, $\mathrm{Tg}$ was acting in a direction opposite to the intended movement. A static extensor torque of $-1.4 \mathrm{Nm}$ was produced prior to movement start and increased to $-2.5 \mathrm{Nm}$ at the time of peak acceleration. Flexion movements made with gravity exhibited an initial agonist burst followed by phasic activity in the antagonist, similar to that previously observed in extension movements performed with gravity. Extension movements made against gravity showed early, simultaneous phasic muscle activity in both the agonist and antagonist, as had been observed in flexion movements against gravity.

\section{Discussion}

Movements made under different gravitational loading conditions were produced by modulation of a basic muscle activation pattern resulting in a common movement profile. This general finding is consistent with that observed by Stein et al. (1988). The studies reported here, however, have demonstrated how the CNS alters the known relationships between phasic muscle activity and specific kinematic parameters, to preserve similar movements under different conditions. For example, subtle differences were apparent in the relationship between AG1 duration and movement amplitude in movements made with gravity. Across a 30 deg range of movement amplitude, AG1 duration increased by $96 \%$ in movements made against gravity. This finding is similar to that reported for movements made in the horizontal plane (Berardelli et al. 1984; Brown and Cooke 1984). In contrast, AG1 burst duration increased by only $29 \%$ in movements made with gravity, across the same amplitude. Recently, Brown and Cooke (1990) have proposed that changes in AG1 are related not to parameters such as movement amplitude but rather to the temporal characteristics of the movement. They showed, for example, that AG1 duration increases with increasing acceleration duration in movements where both movement amplitude and duration are held constant. AG1, in conjunction with ANT, is thought to reg- ulate the rate of increase and decrease of acceleration. In the present task, although the duration of acceleration increased in movements made with and against gravity, the influence of gravitational torque on forearm rotation must be taken into account. During extension movements, the torque due to gravitational acceleration acted in the same direction as the movement. Hence, it is quite possible that the CNS takes advantage of gravitational forces and uses it in combination with muscle activity to produce movement. This would then result in a reduction of the total muscle activity required to accelerate the limb, leading to a relatively small increase in AG1 duration with movement amplitude, compared to movements made against gravity or in the horizontal plane.

Movements made against gravity, although also characterized by time symmetric velocity profiles, were produced by changes in the pattern of phasic muscle activity. Most striking was the phasic antagonist activity which occurred at about the same time as AG1. A second, later burst of antagonist activity commonly occurred. Such early antagonist activity has been previously described (Marsden et al. 1983; Karst and Hasan 1987; Cooke and Brown 1990), but is commonly of rather small amplitude as was observed during extension movements made with gravity in this study. It should be noted that the pattern of muscle activation observed in movements against gravity in this study does not correspond with the findings of Cheron and Godaux (1986), who reported a characteristic 'triphasic' EMG pattern for flexion movements made against gravity. Since they presented no kinematic data, it is difficult to interpret their findings within the context of the present study.

In attempting to understand the role of this early antagonist activity it must first be emphasized that the EMG record is, at best, an indirect representation of the total force produced by the muscle (Loeb and Gans 1986). Thus, although the amplitude of the agonist and antagonist appear to be equal, the agonist must have produced greater force for movement to occur. Keeping this in mind, a number of hypotheses can be considered. One possibility arises when considering the forces acting at the joint during rapid rotation. As the limb moves from one position to another, there is a tendency for the articulating surfaces of the joint to separate as a result of centrifugal forces. A centripetal force is necessary to counteract this force and can be provided either passively by ligaments or by active muscle contraction. Karst and Hasan (1987) have proposed that the antagonist may play a role in this function. They found that during the performance of inertially loaded forearm movements in the horizontal plane, antagonist activity was greater than that required simply for stopping the movement. In addition, many subjects exhibited coactivation of the agonist and antagonist at the end of these movements. They suggested that 'excess' antagonist activity, in conjunction with agonist activity, may serve to provide the force necessary to maintain joint congruency, particularly at high velocities. This hypothesis could 
be extended to the present set of experiments when considering the loads acting at the joint. It is possible that the large torques generated by the muscles to accelerate the limb, in a direction opposing the gravitational torque, increase the likelihood of compromising the joint surface. Coactivation prior to movement onset may be an anticipatory response to prevent this from occurring.

An alternative explanation of the early antagonist activity when moving against gravity is related to shoulder stabilisation. Thus, in Fig. 8 , biceps acts across the shoulder as well as the elbow. A similar explanation might hold for Fig. 5 (flexions against gravity) if the early triceps activity were due to cross talk from the biceps or the long head of triceps (which also acts across the shoulder). Cross talk from biceps appears quite unlikely since in the extension movements of Fig. 5 there is no triceps activity during the late, large biceps activity. In regards to cross talk from lateral triceps, recordings were made from both lateral and long triceps during the course of these experiments (to be reported elsewhere). In brief, during flexion (against gravity) movements, the long head was barely active during the early burst in the lateral head. Thus, the early antagonist burst does not appear to be related to shoulder stabilisation.

Hogan (1984) hypothesized that the CNS controls the activity of the musculoskeletal system by modulating the mechanical impedance of muscle. Using mathematical modelling and experimental manipulation of static arm positions, he showed that when the limb is subjected to destabilizing gravitational torques, the CNS increases joint stability through cocontraction. In the present set of experiments, the magnitude of the gravitational torque at the start of movements, with and against gravity, was approximately the same. However, it is possible that when the limb moves in the same direction as the 'destabilizing' force, there is no need to increase stability at the joint. The limb moves in the direction of the gravitational force partly through generation of active force, and to some extent by succumbing to the 'destabilizing' force. Thus, the force generated by the muscle prior to movement start is relatively small and is reflected in the relatively small change in AG1 duration. In movements against gravity, the situation is more complex. Not only must the CNS generate adequate muscle activity to initiate movement, but it must also ensure that in generating large forces the limb is not subjected to unwanted perturbations (Lestienne et al. 1981). Thus, in this case, the role of the agonist is not simply to regulate acceleration but, in addition, to act with the antagonist to increase joint stiffness, creating a stable base from which to begin movement.

This study has shown that muscle activation patterns in movements performed under different gravitational loads are modified, resulting in the preservation of a common movement trajectory. What is of interest is that the CNS modulates a basic pattern of activation by taking advantage of external forces. It would appear that in planning and coordinating movement the CNS not only exploits the passive, interactive, and mechanical properties of the system (Bernstein 1967), but also utilizes the various 'resources' available in the environment in which the action takes place.

Acknowledgements The authors appreciate the help of Dr. K. Hayes and D. Wolfe. The work reported here was supported by the Natural Sciences and Engineering Research Council of Canada.

\section{References}

Atkeson CG, Hollerbach JM (1985) Kinematic features of unrestrained vertical arm movements. J Neurosci 5:2318-2330

Benecke R, Meinck H-M, Conrad B (1985) Rapid goal-directed elbow flexion movements: limitations of the speed control system due to neural constraints. Exp Brain Res 59:470-477

Berardelli A, Rothwell JC, Day BL, Kachi T, Marsden CD (1984) Duration of the first agonist EMG burst in ballistic movements. Brain Res 304:183-187

Bernstein, N (1967) The coordination and regulation of movements. Pergamon Press, Oxford

Brown SH, Cooke JD (1984) Initial agonist burst duration depends on movement amplitude. Exp Brain Res 55:523-527

Brown SH, Cooke JD (1990) Movement-related phasic muscle activation. I. Relations with temporal profile of movement. J Neurophysiol 63:455-464

Cheron G, Godaux E (1986) Self-terminated fast movement of the forearm in man: amplitude dependence of the triple burst pattern. J Biophys Biomecanique 10:109-117

Cooke JD, Brown SH, Cunningham DA (1989) Kinematics of arm movements in elderly humans. Neurobiol Aging 10:150-165

Gentilucci M, Castiello U, Corradini ML, Scarpa M, Umilta C, Rizzolatti (1991) Influence of different types of grasping on the transport component of prehension movements. Neuropsychologia 29:361-378

Lestienne F, Polit A, Bizzi E (1981) Functional organization of the motor process underlying the transition form movement to posture. Brain Res 230:121-131

Loeb GE, Gans C (1986) Electromyography for experimentalists. The University of Chicago Press, Chicago

Marsden CD, Obeso JA, Rothwell JC (1983) The function of the antagonist muscle during fast limb movements in man. J Physiol (Lond) 335:1-13

Hasan Z (1986) Optimized movement trajectories and joint stiffness in unperturbed, inertially loaded movements. Biol Cybern $53: 373-382$

Hogan N (1984) An organizing principle for a class of voluntary movements. J Neurosci 4:2745-2754

KKaminski T, Gentile AM (1986) Joint control strategies and hand trajectories in multijoint pointing movements. $\mathbf{J}$ Motor Behav 18:261-278

Karst GM, Hasan Z (1987) Antagonist muscle activity during forearm movements under varying kinematic and loading conditions. Exp Brain Res 67:391-401

Morasso P (1981) Spatial control of arm movements. Exp Brain Res 42:223-227

Munhall K.G, Ostry DJ, Parush A (1985) Characteristics of velocity profiles of speech movements. J Exp Psychol 11:457-474

Nelson WL (1983) Physical principles for economics of skilled movements. Biol Cybern 46:135-147

Ostry DJ (1986) Characteristics of human jaw movements in mastication and speech. Neurosci Lett 26:S87

Ostry DJ, Cooke JD, Munhall KG (1987) Velocity curves of human arm and speech movements. Exp Brain Res 68:37-46

Soechting JF (1984) Effect of target size on spatial and temporal characteristics of a pointing movement in man. Exp Brain Res 54:121-132

Stein RB, Cody FW, Capaday C (1988) The trajectory of human wrist movements. J Neurophysiol 59:1814-1830 